\title{
Performance evaluation of blockchains in the internet of things
}

\author{
Tanweer Alam \\ Department of Computer Science, Faculty of Computer and Information Technology, \\ Islamic University of Madinah, Saudi Arabia
}

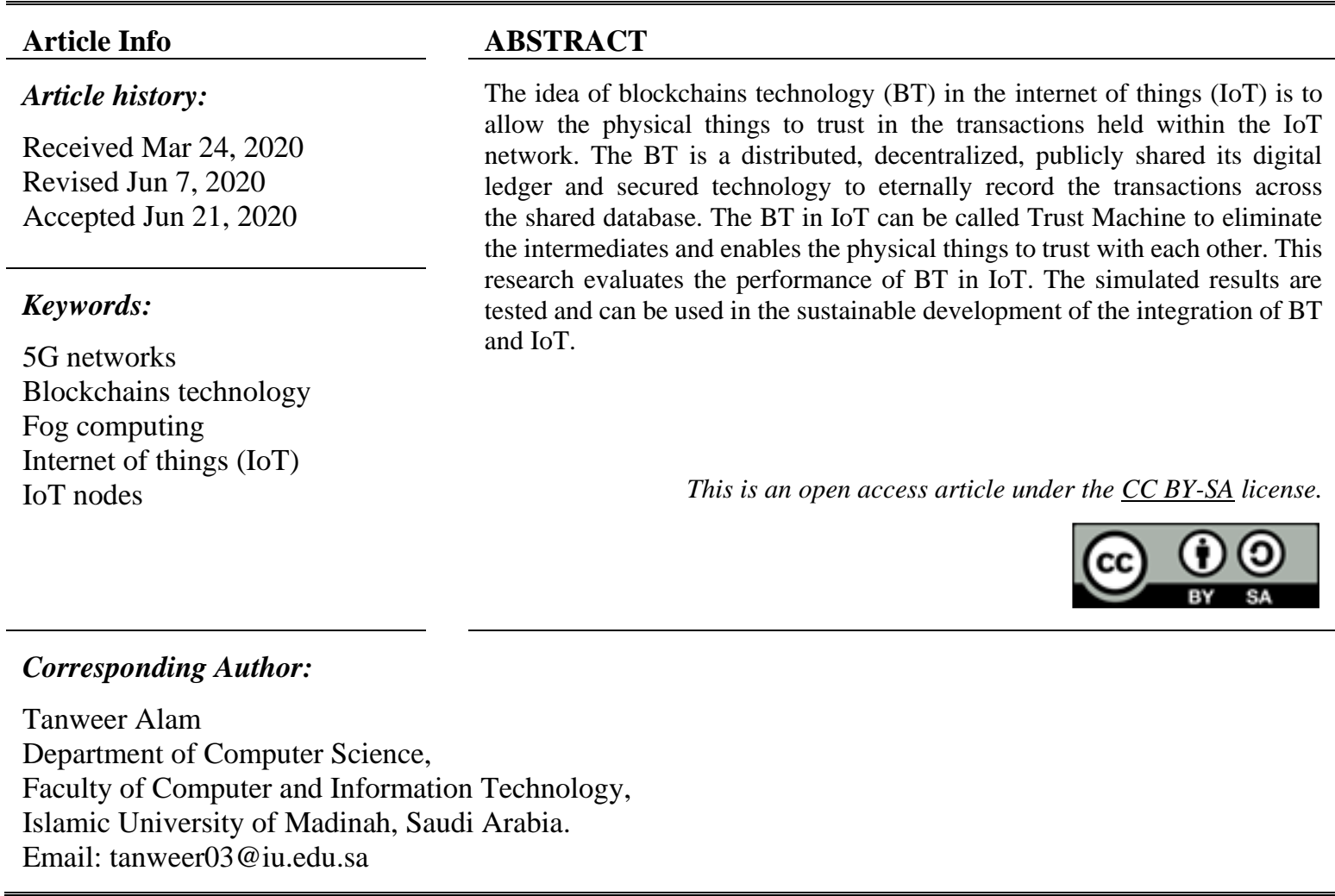

\section{INTRODUCTION}

The internet of things (IoT) enables the physical things to connect, communicate and exchange information without a human to human or human to machine interaction [1]. The blockchains technology (BT) is a growing chain of blocks with the cryptographic hash to eternally record the transactions and store in public database. The IoT and BT both technologies are presumable emerging terms and the framework to integrate these technologies can generate new opportunities in the area of communication security and reliability among internet of physical things [2]. But scalability is a big challenge to integrate both the technologies together because of a huge number of physical connected things in the heterogeneous network [3-6].

The BT in IoT is a novel technology that acts with decentralized, distributed, public and real-time ledger to store transactions among IoT nodes [7]. A blockchain is a series of blocks, each block is linked to its previous blocks [8]. Every block has a cryptographic hash code, previous block hash, and its data. The transactions in BT are the basic units that are used to transfer data between IoT nodes. The IoT nodes are different kind of physical but smart devices with embedded sensors, actuators, programs and able to communicate with other IoT nodes [9].

\section{THE ROLE OF BT IN IOT}

The role of BT in IoT is to provide a procedure to process secured records of data through IoT nodes represented on Figure 1. BT is a secured technology that can be used publicly and openly [10-14]. The IoT requires this kind of technology to communicate securely among the IoT nodes [15-17]. The transactions in 
BT could be traced and explored through anyone who is authenticated to communicate within the IoT. The BT in IoT may help to improve communication security [18']. The major benefits of BT with IoT are:

- $\quad$ Develop trust among IoT public nodes and minify the risk of collisions [19-21],

- Minify the processing costs through direct communication without the third parties [22],

- $\quad$ speed up the transactions in real time.

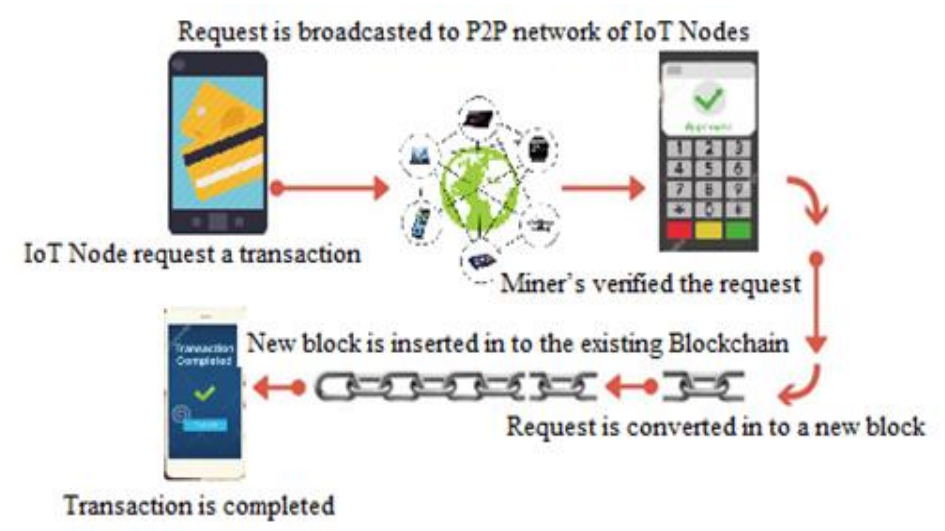

Figure. 1. Transactions Process in BT

\section{RESULTS AND ANALYSIS} formula [8]:

In BT is a series of blocks. The size of a block (BS) in blockchain can be obtained by the following

$\mathrm{BS}($ in $\mathrm{MB})=\mathrm{HS}($ in Bytes $)+\mathrm{TS}($ in Bytes $) *$ No of transactions in that block.

Where HS is the size of the header. The Total Mining energy (Te) can be calculated by the sum of all connected IoT nodes mining energy $(\mathrm{E})$ :

$$
\mathrm{Te}=\mathrm{Te}=\sum_{i=1}^{n} E(i)
$$

Time (t) taken by a block between interconnected IoT nodes with their bandwidth Bw:

$$
\mathrm{t}=\mathrm{BS} / \mathrm{Bw}(\mathrm{i}, \mathrm{j})
$$

where Bw(i,j) is the bandwidth between the IoT nodes i,j.

The framework width (Fw) can be obtained by

$$
\mathrm{Fw}=\operatorname{Max}(\mathrm{t})
$$

The hash is the crypto code of the previous block $(\mathrm{Hc})$ that is assigned to every block in the blockchain. The block occurrence (Bo) can be obtained by

$$
\mathrm{Bo}=\mathrm{Te} / \mathrm{Hc} \text {. }
$$

The transactions per second (Ts) held in the blockchain can be obtained by the formula:

$$
\mathrm{Ts}=\mathrm{Bo} * \text { No of transactions in that block }
$$

The fork in blockchain can happen if the miners received the blocks at the same time. The probability of blockchain fork $\mathrm{P}(\mathrm{F})$ can be calculated by the formula:

$$
\mathrm{P}(\mathrm{F})=1-(1+\mathrm{Bo} * \mathrm{Fw}) * \mathrm{e}-\mathrm{Bo} * \mathrm{Fw}
$$


Consider the newly created block BN that is referenced by two previously created blocks. The BN is placed between these two blocks. Consider that the block BN is verified by the miners, the time $\mathrm{BN}(\mathrm{t})$ is represented the verification time calculated by the Poisson process $\mu$ [23-25]. Suppose $\alpha$ be the time taken by the IoT node to compute the transaction process to the fog network. So, the total time taken by each transaction to be visible on the network is $\alpha+\mathrm{t}$ [9]. Consider that $\mathrm{N}$ is the total number of unverified blocks [26-27]. The probability of $\mathrm{BN}$ in the blockchain of the IoT-Fog framework is calculated by the following formula.

$$
\mathrm{P}(\mathrm{BN})=\mathrm{N} /(\mathrm{N}+\mu \alpha)
$$

Consider the 5 IoT nodes (Figure 2) connected with the P2P network with the mining energy $500,1000,300,700$, and 1500 respectively. They are able to do transactions in the proposed framework. Suppose the size of the header is 100 bytes and the size of the transaction is 500 bytes. The IoT nodes in the framework are five. Using (1), we have calculated the block size. BS (in MB) $=$ HS (in Byes) + TS (in Bytes) * No of transactions in that block BS=100+500*5=2600 MB

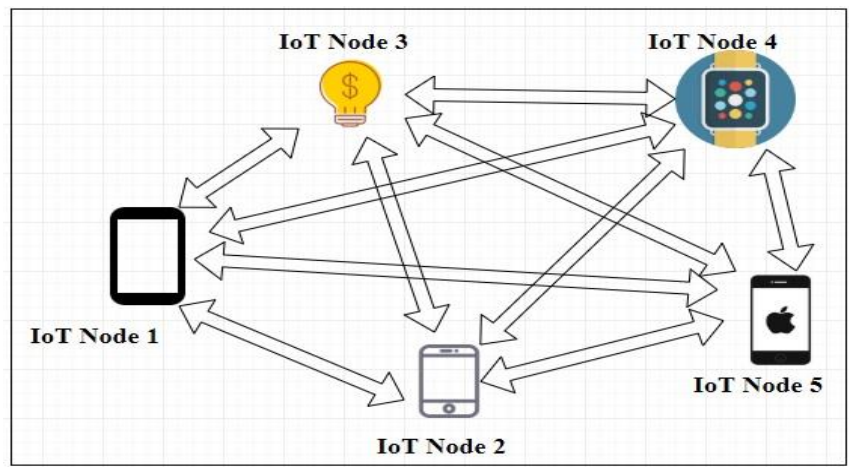

Figure 2. IoT Nodes connected in the P2P network

By using (2), we have calculated the total mining energy

$$
\begin{aligned}
\mathrm{Te} & =\sum_{i=1}^{n} E(i) \\
& =500+1000+300+700+1500 \\
& =4000
\end{aligned}
$$

Suppose BS=1 MB= 1,048,576 Bytes

Total number of transactions in a block $=(\mathrm{BS}-\mathrm{HS}) / \mathrm{TS}$

$$
\begin{aligned}
& =1,048,576-100 / 500 \\
& =2096.952
\end{aligned}
$$

Now, according to (3), we have to calculate the bandwidth of the framework as shown in Table 1.

Table 1. Bandwidth in the framework device by device

\begin{tabular}{cccccc}
\hline $\mathrm{x}$ & IoT Node 1 & IoT Node 2 & IoT Node 3 & IoT Node 4 & IoT Node 5 \\
\hline IoT Node 1 & $\mathrm{x}$ & $1 / 8$ & $1 / 9$ & $1 / 5$ & $17 / 86$ \\
IoT Node 2 & - & $\mathrm{x}$ & $1 / 5$ & $15 / 79$ & $1 / 9$ \\
IoT Node 3 & - & - & $13 / 40$ & $17 / 71$ & $13 / 40$ \\
IoT Node 4 & - & - & - & $\mathrm{x}$ & $1 / 9$ \\
IoT Node 5 & - & - & - & - & $\mathrm{x}$ \\
\hline
\end{tabular}

The IoT-Fog framework is tested using 5 local IoT nodes and 2 remote IoT nodes. The NoSQL database software is used to store the records fetched from blockchain in the fogging by the tool named OPENSHIFT platform. Cloud is created by Amazon, bandwidth of the system is shown in Figure 3. The FogSim software is used to connect the IoT devices to the fogging. Performance evaluation of IoT nodes in the IoT-Fog is represented in Figure 4. The results are found in Table 2. 


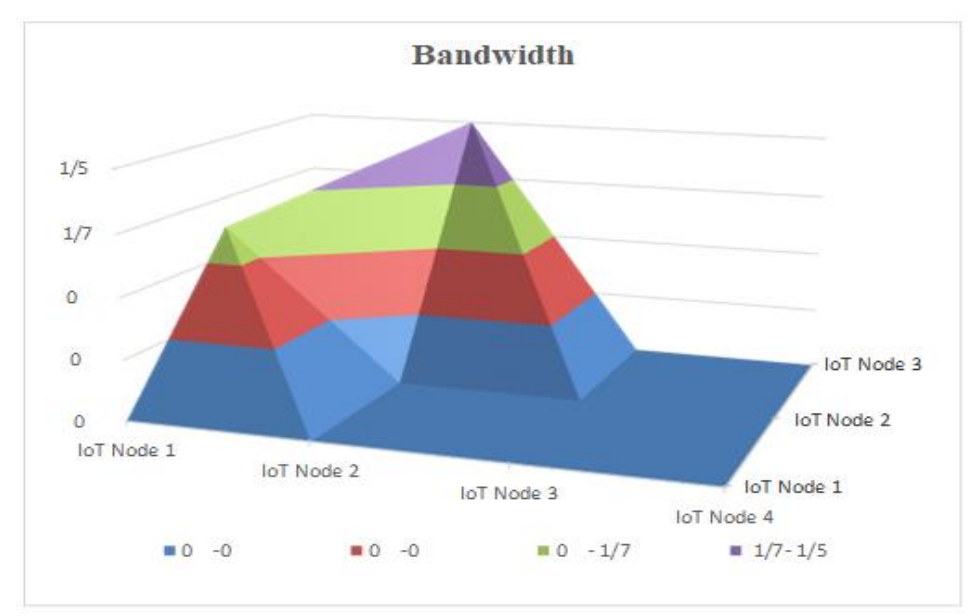

Figure 3. Bandwidth of the system

Table 2. Performance evaluation of IoT nodes in the IoT-Fog

\begin{tabular}{llllll}
\hline & IoT Node 1-Fog & IoT Node 2-Fog & IoT Node 3-Fog & IoT Node 4-Fog & IoT Node 5-Fog \\
\hline Delay by IoT Node & 5.16548 & 5.16548 & 5.16548 & 5.16548 & 5.16548 \\
Delay by Network & 3.2111 & 3.2111 & 3.2111 & 3.2111 & 3.2111 \\
Node Energy & $1.29 \mathrm{E}+007$ & $1.30 \mathrm{E}+007$ & $1.31 \mathrm{E}+007$ & $1.31 \mathrm{E}+007$ & $1.33 \mathrm{E}+007$ \\
Mobility Energy & 3267871.04 & 3267189.177 & $1.33 \mathrm{E}+007$ & $2.91 \mathrm{E}+007$ & $5.52 \mathrm{E}+007$ \\
Fog Energy & 998835.435423 & 2097670.862 & 4195341.724 & 8390683.448 & $1.68 \mathrm{E}+007$ \\
Execution Time & 1672 & 1983 & 2864 & 4972 & 8943 \\
Network Usage & 12078.45 & 22301.89 & 39871.78 & 88729.90 & 172987.34 \\
\hline
\end{tabular}

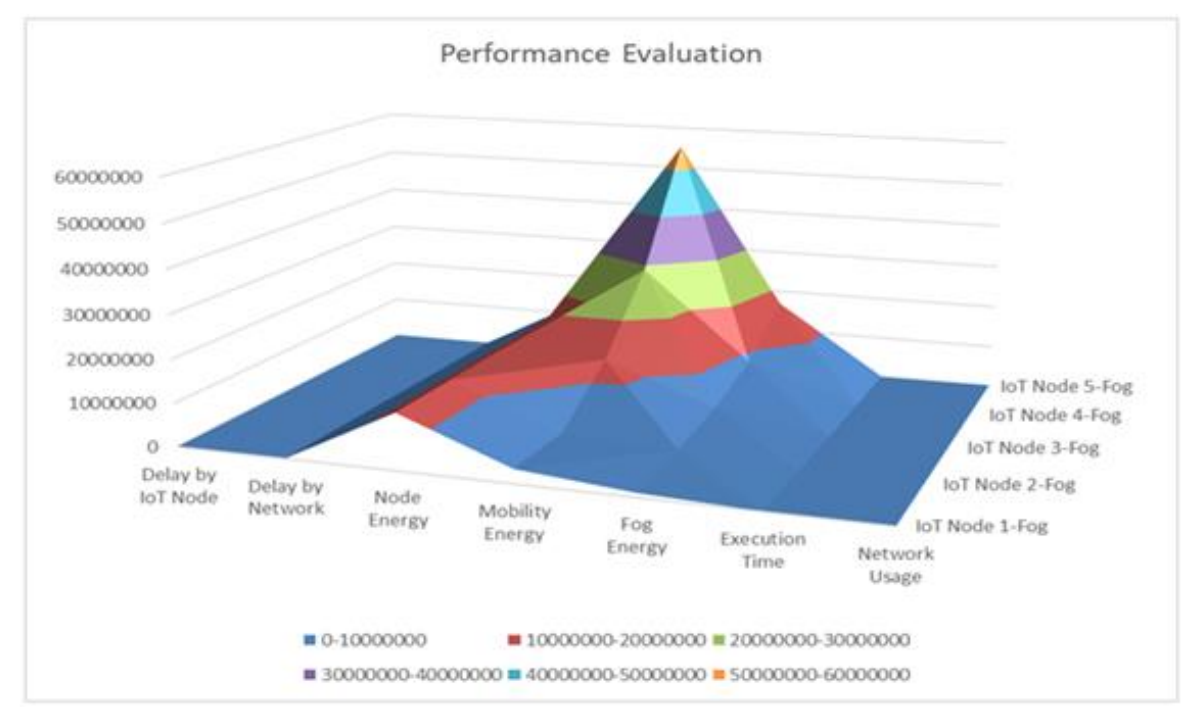

Figure 4. Performance evaluation of IoT nodes in the IoT-Fog

\section{CONCLUSION}

The proposed research is the step forward in the area of internet of things and blockchain technology. The middleware framework is implemented and tested in the virtually created heterogeneous environment of the $5 \mathrm{G}$ network. The data of the secured transactions are stored in the blocks of the blockchain. The verified blocks by the miners are only entered into the blockchain network and linked to the blockchain by using the secured encrypted hash code. In the performance evaluation of the framework, all the possible factors such as Delay by IoT Node, Delay by Network, Node Energy, Mobility Energy, Fog Energy, Execution Time and Network Usage are evaluated. 


\section{REFERENCES}

[1] Alaba, F. A., Othman, M., Hashem, I. A. T., Alotaibi, F. "Internet of Things security: A survey." Journal of Network and Computer Applications, 88, 10-28, 2017.

[2] Dorri, Ali, Salil S. Kanhere, Raja Jurdak. "Towards an optimized blockchain for IoT." Proceedings of the Second International Conference on Internet-of-Things Design and Implementation. ACM, 2017.

[3] Alam, Tanweer, and Mohamed Benaida. "CICS: Cloud-Internet Communication Security Framework for the Internet of Smart Devices." International Journal of Interactive Mobile Technologies (IJIM), vol. 12, no. 6, pp. 74-84, 2018.

[4] Shanti Bruyn, "Blockchain an introduction", research report, University Amsterdam, 2017

[5] Florea BC. "Blockchain and Internet of Things data provider for smart applications." In 2018 7th Mediterranean Conference on Embedded Computing (MECO), pp. 1-4, 2018.

[6] Alam, T. "Cloud Computing and Its Role in the Information Technology." IAIC Transactions on Sustainable Digital Innovation (ITSDI), vol. 1, no. 2, pp. 108-115, 2020.

[7] Conoscenti, Marco, Antonio Vetro, and Juan Carlos De Martin. "Blockchain for the Internet of Things: A systematic literature review." Computer Systems and Applications (AICCSA), 2016 IEEE/ACS 13th International Conference of. IEEE, 2016.

[8] Kouzinopoulos, Charalampos S., et al. "Using blockchains to strengthen the security of internet of things." International ISCIS Security Workshop. Springer, Cham, 2018.

[9] Alam T, Benaida M. "The Role of Cloud-MANET Framework in the Internet of Things (IoT)." International Journal of Online and Biomedical Engineering (IJOE), vol. 14, no. 12, pp. 97-111, 2018.

[10] Alam, Tanweer, and Mohammed Aljohani. "An approach to secure communication in mobile ad-hoc networks of Android devices." In 2015 International Conference on Intelligent Informatics and Biomedical Sciences (ICIIBMS), IEEE, pp. 371-375, 2015.

[11] Alam, Tanweer, and Mohammed Aljohani. "Design a new middleware for communication in ad hoc network of android smart devices." In Proceedings of the Second International Conference on Information and Communication Technology for Competitive Strategies. ACM, p. 38, 2016.

[12] Alam, Tanweer, and Mohammed Aljohani. "Design and implementation of an Ad Hoc Network among Android smart devices." In Green Computing and Internet of Things (ICGCIoT), 2015 International Conference on, IEEE, pp. 1322-1327, 2015.

[13] Alam, Tanweer. "Fuzzy control based mobility framework for evaluating mobility models in MANET of smart devices." ARPN Journal of Engineering and Applied Sciences, vol. 12, no. 15, pp. 4526-4538, 2017.

[14] Alam, Tanweer. "Middleware Implementation in Cloud-MANET Mobility Model for Internet of Smart Devices." International Journal of Computer Science and Network Security, vol. 17, no. 5, pp. 86-9, 2017.

[15] Alam, Tanweer. "A reliable framework for communication in internet of smart devices using IEEE 802.15.4." ARPN Journal of Engineering and Applied Sciences, vol. 13, no. 10, pp. 3378-3387, 2018.

[16] Baha Rababah, Tanweer Alam, Rasit Eskicioglu. "Next Generation Internet of Things Architecture towards Distributed Intelligence: Reviews, Applications, and Research Challenges." Journal of Telecommunication, Electronic and Computer Engineering, vol. 12, no. 2, 2020.

[17] T. Alam. "Design a blockchain-based middleware layer in the Internet of Things Architecture." JOIV: International Journal on Informatics Visualization, vol. 4, no. 1, Feb. 2020.

[18] Lee, Boohyung, and Jong-Hyouk Lee. "Blockchain-based secure firmware update for embedded devices in an Internet of Things environment." The Journal of Supercomputing, vol. 73, no. 3, pp. 1152-1167, 2017.

[19] Tanweer Alam. "A Reliable Communication Framework and Its Use in Internet of Things (IoT)." International Journal of Scientific Research in Computer Science, Engineering and Information Technology (IJSRCSEIT), vol. 3 , no. 5, pp. 450-456, May-June.2018.

[20] Tanweer Alam, "5G-Enabled Tactile Internet for smart cities: vision, recent developments, and challenges." Jurnal Informatika, Vol. 13, No 2, pp. 1-10, July 2019.

[21] Tanweer Alam. "A Middleware Framework between Mobility and IoT Using IEEE 802.15.4e Sensor Networks." Jurnal Online Informatika, Vol 4, No 2, pp. 90-94, 2019.

[22] Tanweer Alam. "Blockchain and its Role in the Internet of Things (IoT)." International Journal of Scientific Research in Computer Science, Engineering and Information Technology, vol. 5, no. 1, pp. 151-157, 2019.

[23] Tanweer Alam. "Efficient and Secure Data Transmission Approach in Cloud-MANET-IoT integrated Framework." Journal of Telecommunication, Electronic and Computer Engineering (JTEC), Vol. 12 No. 1, 2020.

[24] Tanweer Alam. "Internet of Things: A Secure Cloud-Based MANET Mobility Model." International Journal of Network Security, Vol. 22, No. 3, pp. 514-520, 2020.

[25] Tanweer Alam. "IoT-Fog: A Communication Framework using Blockchain in the Internet of Things." International Journal of Recent Technology and Engineering (IJRTE), vol. 7, no. 6, pp. 833-838, 2019.

[26] Tanweer Alam. "Middleware implementation in MANET of Android Devices." International Journal of Electronics and Information Engineering, vol. 12, no. 2, 2020.

[27] Tanweer Alam, Baha Rababah. "Convergence of MANET in Communication among Smart Devices in IoT." International Journal of Wireless and Microwave Technologies (IJWMT), Vol.9, No.2, pp. 1-10, 2019. 